\title{
Planning the Base Station Layout in UMTS Urban Scenarios: A Simulation Approach to Coverage and Capacity Estimation $^{1}$
}

\author{
Enrica Zola and Francisco Barceló \\ Universitat Politècnica de Catalunya \\ c/ Jordi Girona 1-3, 08034 Barcelona (Spain) \\ enrica@entel.upc.es, barcelo@mat.upc.es
}

\begin{abstract}
This paper analyzes the performance of a base station layout for a UMTS network in a densely populated city. The study is carried out using snapshot simulations of an actual city (Barcelona, Spain) with specific traffic and propagation profiles. A first layout is proposed in order to guarantee good coverage with a minimum number of base stations. This layout is approached taking into account the link budget analytical calculations and the first simulation results for a single-cell environment. A second layout is then presented in an attempt to solve the problems that arise in the first (e.g. shadows, blind zones, etc.). This is carried out by finely tuning parameters from the first layout, adding new base stations and changing the locations of the existing ones.
\end{abstract}

\section{Introduction}

A new feature of UMTS is higher user bit rates: $384 \mathrm{kbps}$ on Circuit-Switched (CS) connections, and up to 2 Mbps on Packet-Switched (PS) connections. At the beginning of the UMTS era, most of the traffic will be voice, but the share of data will grow progressively. It is necessary to study the $3 \mathrm{G}$ radio network planning carefully, in order to fulfill the requirements for coverage, capacity and quality of service [1].

Planning the Base Station (BS) layout in a city is a complex task since there are many constraints that make it difficult to locate antennas in the desired positions. This paper presents an approach to the layout problem by positioning BSs according to the Link Budget (LB) calculations carried out for the city of Barcelona. In a second step, the specific problems that arise from the first plan are taken into account. Only a long process of trial-and-error, which is beyond of the scope of this paper, can lead to an optimum trade-off between full coverage and low investment in BSs.

The goal of this study is to show the basics of the aforementioned trial-and-error process. Although only two steps are carried out in the paper due to space constraints, this two-stepsonly approach gives good results. With regard to capacity planning, simulations show how a slight improvement can be obtained by finely balancing the power share in the downlink (DL). However, the benefit obtained in terms of coverage does not carry through in terms of capacity.

The paper is structured as follows. In Section 2, we describe the LB evaluation process. Section 3 introduces the snapshot simulation tool that was used to analyze the network. The simulator is used to verify the coverage and capacity results presented in Section 2. According to the results, the first BSs location plan for the city of Barcelona is drawn in Section 4. Slight modifications to the first plan improve the overall coverage greatly, while capacity problems relating to DL power sharing and interference cannot be solved.

\footnotetext{
${ }^{1}$ This research has been funded by the Spanish Ministry of Science and Technology through CICYT project RUBI 2003-01748
} 


\section{Analytical Evaluation of the Link Budget}

This section presents UMTS radio network planning including capacity and coverage planning $[2,3]$. In the dimensioning phase, the number of BS sites is estimated on the basis of the operator's requirements for coverage, capacity and quality of service. Capacity and coverage are closely related in W-CDMA networks [4] and must be considered simultaneously in the study.

\subsection{Coverage Plan}

In order to guarantee sufficient coverage and to limit costs by using the lowest possible number of BSs, it is important to position BSs at an optimal distance from each other. One can estimate this distance by computing the maximum losses that the UMTS signal suffers. This first approach to radio network planning is based on the analytical estimation of radio signal propagation. This process is known as the link budget [5,6] and is independent from the simulation results presented in the following sections. Note that the simulation tool carries out its own calculations by means of propagation models that are better suited to the environment analyzed, and are hence more complex. The LB estimation is carried out using the OkumuraHata propagation model, in which the propagation losses $\left(L_{O H}\right)$ in an urban scenario are computed as:

$$
L_{O H}=133.76+34.79 \log d,
$$

where $d$ is the distance in kilometers. More details can be found in [7, 8].

Table 1 shows the cell coverage ranges (i.e. distance in meters) obtained from the uplink (UL) budget for an urban environment. A similar calculation is obtained (although it is not displayed in this paper) for different system loads and for different environments, since both have an impact on the cell range. Other parameters are set by the standard, e.g. the maximum power a Mobile Station (MS) can transmit depends on its class. The standard classifies four types of MSs, but Class 4, for which the maximum power is limited to $21 \mathrm{dBm}$, will be most used. Class 3 will be used for high data rate transmissions ( $384 \mathrm{kbps})$ only. Moreover, the bit rate of the communication directly settles the SIR target at the receiver and, consequently, has an impact on the sensibility of the BS. The fading margin groups a set of margins: log-normal and Rayleigh fading, soft handover and diversity gain, power-control, etc.

Table 1. Results from the link budget in an urban environment (uplink)

\begin{tabular}{lcccc}
\hline \multicolumn{5}{c}{ Total system load: 60\% } \\
\hline & Urban environment - Pedestrian user \\
\hline MS tx power $\left(P_{t x}\right)[\mathrm{dBm}]$ & 21 & 21 & 21 & 24 \\
BS noise power $[\mathrm{dBm}]$ & -101.2 & -101.2 & -101.2 & -101.2 \\
Bit rate $[\mathrm{kbps}]$ & 12.2 & 64 & 144 & 384 \\
Sensibility $(S)[\mathrm{dBm}]$ & -122.5 & -115.6 & -112.7 & -109.6 \\
Total losses $(L)[\mathrm{dB}]$ & 4 & 1 & 1 & -1 \\
Fading margin $(f m)[\mathrm{dB}]$ & 3 & 3 & 3 & 3 \\
Propagation losses $\left(P_{t x}-S-L-f m\right)[\mathrm{dB}]$ & 136.46 & 132.6 & 129.7 & 131.58 \\
Cell coverage range $[\mathrm{m}]$ & 1196 & 924 & 764 & 866 \\
\hline
\end{tabular}

It can be observed that, when considering a service with a higher data rate, the cell coverage decreases, unless a higher transmission power is used as in CS384. Depending on the services to be provided, the operator must establish minimum cell coverage (i.e. the coverage for the most constraining service) required in order to guarantee good coverage to every user. In the $\mathrm{DL}$, the transmission power of the BS $(20 \mathrm{~W})$ is shared between all users. As higher bit rate communications need more power than low rate ones, the coverage range is highly sensitive to 
the cell load: the UL is coverage-limited (i.e. distance from the BS) while the DL is capacitylimited.

\subsection{Capacity Dimensioning}

W-CDMA systems are interference-limited: the link performance depends on the ability of the receiver to discern signal in presence of interference. The LB displayed in Table 1 is valid for $60 \%$ load only: in general, the coverage range depends on the cell load. It represents the capacity of the system to carry a specific traffic load (depending on the density of traffic and on the kind of services considered). In [3], the authors present simulations with different traffic densities for a single BS environment that show how coverage decreases when load increases.

The second phase of dimensioning is estimating the amount of traffic supported per BS: we have to find an agreement between the maximum load inside a cell (i.e. the maximum number of simultaneous users for each service) and the number of traffic channels allocated to the BS [9]. In this process, only CS traffic has been taken into account, since PS services do not need circuit reservation. By estimating the traffic carried with a given $\mathrm{GoS}$, which is represented here by the blocking probability, one could visualize PS services being provided during idle periods of CS traffic.

Cell ranges for each environment and load are estimated in the UL budget. We must ensure that the BSs DL power can be shared between all users and that this leaves a sufficient margin for efficient performance.

\section{The Simulation Tool}

The simulation program performs coverage analysis from a set of antennas in a specific traffic and propagation environment, from which it is possible to determine the coverage area of the layout under study. It also performs analysis of the traffic carried on maps that can be shaped by the user (i.e. the kind of services offered and traffic density for each service). By analyzing iterative snapshots of the system, the tool evaluates the average percentage of calls that the system can handle simultaneously.

\subsection{Coverage Analysis}

By working with propagation models that can be molded to the scenario, the simulator estimates the losses and generates a coverage map. In this way, it is possible to compute the BS coverage ranges by simulation [10]. In [3], the authors presented the propagation model used for these simulations. It is an adaptation of the classic Okumura-Hata model in which the parameters are tuned to the specific scenario and topography characteristics of Barcelona. By performing propagation analysis, the simulator can draw maps in which colors represent the signal strength around the BS.

Fig. 1 shows the coverage map around a tri-sectorial BS in the city of Barcelona. Black represents the signal of a power strength under $-90 \mathrm{dBm}$. This corresponds to the out-ofcoverage area for this BS. Dark grey is where the strength remains between -90 and $-80 \mathrm{dBm}$. This area extends to the bottom right-hand side, where the sea begins. As the signal moves away from the BS, it finds obstacles which can weaken its strength. We must determine the limits of the power strength in order to receive the communication correctly (i.e. signal-tointerference target). 


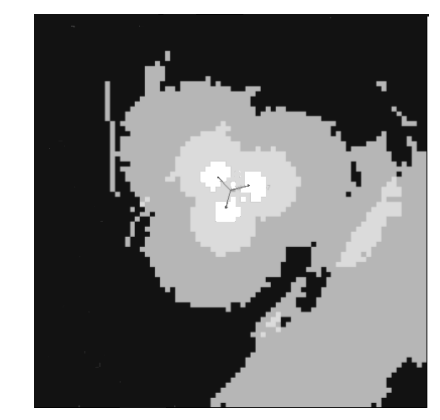

Fig. 1. Coverage Map for a Tri-sectorial BS

\subsection{Traffic Analysis}

The tool simulates a real environment in which each BS serves a specific number of users that request different services. It is possible to work with traffic maps in which the user specifies the Erlang quantity for each service and area. Table 2 shows the values that are commonly assumed in this kind of analysis.

Table 2. Traffic density for different environments

\begin{tabular}{lc}
\hline Environment & Traffic density in the busy hour $\left(\mathbf{E} / \mathbf{k m}^{2}\right)$ \\
\hline Dense urban & $10-90$ \\
Urban & $1-30$ \\
Suburban & $0.5-5$ \\
Vehicular & It depends on the environment \\
Rural & $0-0.3$ \\
\hline
\end{tabular}

\subsection{Monte Carlo Simulation}

The simulator, based on the Monte Carlo approach, relies on the analysis of different snapshots of the system for a variety of conditions. For each snapshot, a number of users with various characteristics are planned in the system and randomly allocated throughout the area analyzed (with a uniform space distribution). Once the system has reached the desired convergence in terms of blocking probability (i.e. the number of dropped calls in the actual iteration converges to the average of all iterations), statistics and pictures describing the system are generated, since it is assumed that enough snapshots have been run in order to give a good statistical representation [2].

\section{BS Layout in the City of Barcelona}

When planning the BSs layout in a real environment, it is important to know the coverage that a BS can provide in order to establish the optimum distances between sites. The authors first ran simulations in a single tri-sectorial site in order to check that the results from the analytical LB of Section 2 were accurate. The results were presented in [3]. There is a good agreement between the cell range simulation and the values listed in Table 1. The multiple service case (i.e. voice and data users at all bit rates) was also analyzed. An important result is that high data rate services increase cell load and tend to decrease the GoS. In this paper, we present the results obtained when simulating a complete BS layout in Barcelona. Coverage problems can be solved by changing the layout, while capacity problems remain. 


\subsection{The First Layout}

In the first plan, a regular pattern was followed when positioning BSs: the underlying is hexagonal with some necessary adjustments. Fig. 2 displays the position of the BSs in the city. Only two services, voice and CS64, were considered. The latter has a smaller coverage range, so this distance (i.e. the one for the most constraining service) was used in the plan. The area analyzed corresponds to the central area of the city of Barcelona, in which two different environments can be found: dense urban, corresponding to the center of the city, and urban, corresponding to the residential areas. In the first zone, we placed a greater number of BSs in order to carry a higher density of traffic. The related coverage map in Fig. 3 shows the signal strength for each point of the city. The lower right-hand side is where the sea begins (see Fig. 2 ), which is why there is good coverage even though there are no sites.

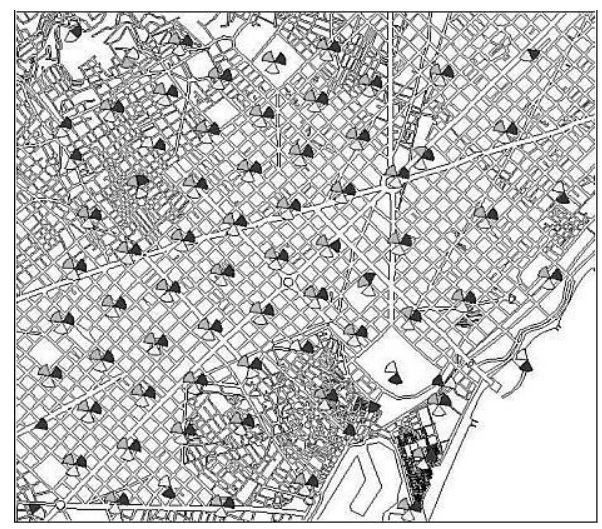

Fig. 2. First BSs layout plan for Barcelona

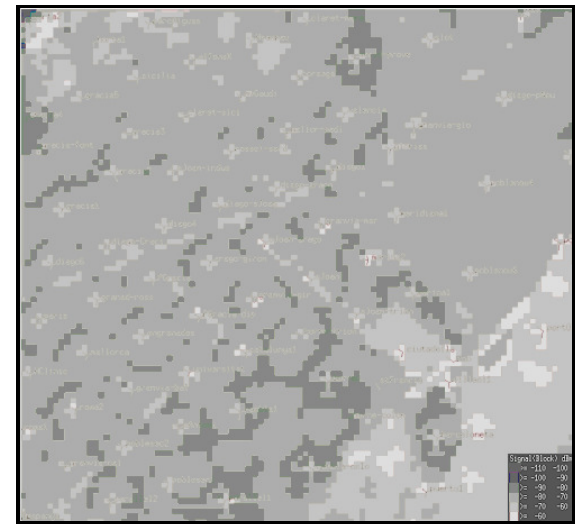

Fig. 3. Coverage map for the first BSs plan

The next step in the study is to analyze the system when giving service to voice users only. Table 3 shows the parameters used in this first simulation. The number of traffic channels is computed according to Erlang-B [11], in order to limit the GoS to 2\%. An extra $30 \%$ of the channels is allocated for handover purposes only.

Table 3. Parameters for the first simulation

\begin{tabular}{ccc}
\hline Environment & Dense urban & Urban \\
\hline Users & Voice & Voice \\
Offered traffic & $40 \mathrm{E} / \mathrm{km}^{2}$ & $20 \mathrm{E} / \mathrm{km}^{2}$ \\
Total area & $11.28 \mathrm{~km}^{2}$ & $9.45 \mathrm{~km}^{2}$ \\
Traffic channels & \multicolumn{2}{c}{65 for each BS } \\
\hline
\end{tabular}

The report provided by the simulator in Table 4 shows that $97.7 \%$ of users can be served. 99.9\% of all dropped users (i.e. 2.3\% of the users attempted) is due to the Mobile ERP Limit (Equivalent Radiated Power). These users are concentrated in areas where coverage is not good. A negligible quantity of users cannot be served due to the CPICH Power Limit (Common PIlot CHannel). These users cannot receive the beacon sequences transmitted by the BSs correctly. In this first analysis, the limited power of the BSs can be efficiently shared between all users (i.e. 0\% is dropped due to DPCH Power Limit, Dedicated Physical CHannel). The simulator allows the user to set the power limits for both the CPICH and DPCH, according to the recommendations given by the operator (in Section 4.2, an example of tuning these values is provided). 
Table 4. Report for voice traffic (first layout)

\begin{tabular}{|c|c|c|c|}
\hline \multicolumn{4}{|c|}{ User type: Voice at $12.2 \mathrm{kbps}$} \\
\hline \multicolumn{4}{|c|}{ Users attempted: 637.9; Users served: 623.4 (97.7\%); Total dropped users: $2.3 \%$} \\
\hline & Reason & No. of users & $\%$ of users (of the $2.3 \%$ / of the total) \\
\hline 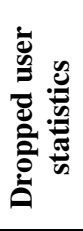 & $\begin{array}{l}\text { Mobile ERP limit } \\
\text { Noise rise limit } \\
\text { Primary CE limit } \\
\text { CPICH power limit } \\
\text { DPCH power limit } \\
\text { Code limit }\end{array}$ & $\begin{array}{c}14.4 \\
0 \\
0 \\
0 \\
0 \\
0\end{array}$ & $\begin{array}{c}99.9 / 2.3 \\
0 \\
0 \\
0.1 / 0 \\
0 \\
0\end{array}$ \\
\hline & Type of HO & No. of users & $\%$ of users \\
\hline 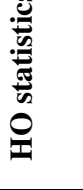 & $\begin{array}{l}\text { Not in HO } \\
\text { Softer HO } \\
\text { Soft HO } \\
\text { Soft-soft HO } \\
\text { Softer-soft HO }\end{array}$ & $\begin{array}{c}182.1 \\
2.0 \\
128.8 \\
303.8 \\
6.6\end{array}$ & $\begin{array}{c}29.2 \\
0.3 \\
20.7 \\
48.7 \\
1.1\end{array}$ \\
\hline
\end{tabular}

The simulator analyzes snapshots of the system. By evaluating the power levels at each point, it can estimate the percentage of users that remain in one of the different handover (HO) situations. A high proportion of users $(48.7 \%$ ) is in a soft-HO with three different BSs (soft-soft $H O$ ); $29.2 \%$ is not in an $\mathrm{HO}$, while $20.7 \%$ is in an $\mathrm{HO}$ with two BSs (soft HO). Small percentages of users remain in other soft-HO situations: $0.3 \%$ in softer $\mathrm{HO}$, which is an $\mathrm{HO}$ involving two sectors of the same BS; $0.9 \%$ and $0.1 \%$ are in $\mathrm{HO}$ with two sectors of the same $\mathrm{BS}$ and another BS.

The next case study consists in the addition of 10\% of CS64 data traffic. The parameters are shown in Table 5.

Table 5. Parameters for the mixed-traffic case study

\begin{tabular}{lcccc}
\hline Environment & \multicolumn{2}{c}{ Dense urban } & \multicolumn{2}{c}{ Urban } \\
\hline Users & Voice & CS 64 & Voice & CS 64 \\
Offered traffic & $40 \mathrm{E} / \mathrm{km}^{2}$ & $4 \mathrm{E} / \mathrm{km}^{2}$ & $20 \mathrm{E} / \mathrm{km}^{2}$ & $2 \mathrm{E} / \mathrm{km}^{2}$ \\
Total area & \multicolumn{1}{c}{$11.28 \mathrm{~km}^{2}$} & \multicolumn{2}{c}{$9.45 \mathrm{~km}^{2}$} \\
Traffic channels & \multicolumn{3}{c}{78 for each BS } \\
\hline
\end{tabular}

Table 6. Report for mixed-traffic (first layout)

\begin{tabular}{|c|c|c|c|}
\hline \multicolumn{4}{|c|}{ User type: Voice and CS data at $64 \mathrm{kbps}$} \\
\hline \multicolumn{4}{|c|}{ Users attempted: 702.1; Users served: 654 (93.1\%); Total dropped users: 6.9\% } \\
\hline & Reason & No. of users & $\%$ of users (of the $6.9 \%$ / of the total) \\
\hline 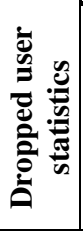 & $\begin{array}{l}\text { Mobile ERP limit } \\
\text { Noise rise limit } \\
\text { Primary CE limit } \\
\text { CPICH power limit } \\
\text { DPCH power limit } \\
\text { Code limit }\end{array}$ & $\begin{array}{c}32.9 \\
0 \\
0 \\
2.2 \\
13.1 \\
0\end{array}$ & $\begin{array}{c}68.4 / 4.72 \\
0 \\
0 \\
4.5 / 0.3 \\
27.2 / 1.88 \\
0\end{array}$ \\
\hline & Type of HO & No. of users & $\%$ of users \\
\hline 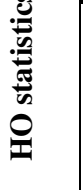 & $\begin{array}{l}\text { Not in HO } \\
\text { Softer HO } \\
\text { Soft HO } \\
\text { Soft-soft HO } \\
\text { Softer-soft HO }\end{array}$ & $\begin{array}{c}187.8 \\
1.9 \\
131.2 \\
324.8 \\
8.3\end{array}$ & $\begin{array}{c}28.7 \\
0.3 \\
20.1 \\
49.7 \\
1.2\end{array}$ \\
\hline
\end{tabular}

Table 6 reports a reduction to $93.1 \%$ of calls attended. The percentage of non-served calls due to coverage problems increases to the $4.7 \%$ of the users attempted. By simply adding a small percentage of data service, problems that are not directly related to the coverage range 
arise. About $1.88 \%$ of the users attempted fail due to the DPCH Power Limit, while $0.3 \%$ cannot be attended due to the CPICH Power Limit. A large proportion of users (49.7\%) stays in an $\mathrm{HO}$ involving three $\mathrm{BSs} ; 28.7 \%$ are not in an $\mathrm{HO}$, and $20.1 \%$ remain in a soft-HO with two different BSs.

\subsection{The Second Layout}

The second layout (see Fig. 4) is an attempt to improve the system's performance when combining voice and CS64 data traffic. The coverage problems in the first plan were solved by tuning down-tilt and orientation of antennas, and by adding new BSs when necessary.
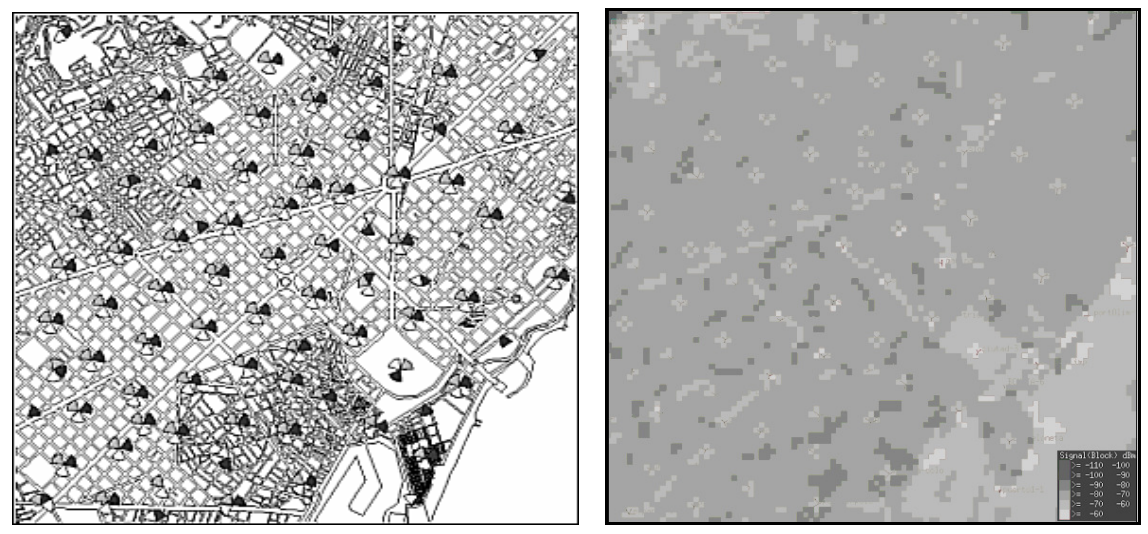

Fig. 4. Second BSs layout plan Fig. 5. Coverage map for the second BSs layout plan

Fig. 5 shows that signal strength improves with respect to Fig. 3 in most of the city area. With this new layout, voice and CS64 data services can be provided to $94.9 \%$ of the users (Table 7). The simulator reports that coverage problems were reduced (dropped calls due to the Mobile ERP Limit are now $0.4 \%$ of the dropped users, which in turn represent $5.1 \%$ of all attempted users), while problems with power sharing in the DL cannot be solved by merely changing the parameters of the antennas. Table 7 reports that $4.5 \%$ of users $(88.8 \%$ of all dropped users) are dropped due to the $\mathrm{DPCH}$ power limit. This simulation also shows that a high proportion of users (55.1\%) are in a soft-HO with three different BSs; $24.1 \%$ are not in an $\mathrm{HO}$, while $18.7 \%$ are in a soft-HO with two BSs.

Table 7. Report for mixed-traffic (second layout)

\begin{tabular}{|c|c|c|c|}
\hline \multicolumn{4}{|c|}{ User type: Voice and CS data at $64 \mathrm{kbps}$} \\
\hline & Jsers attempted: 699. & sers served: 66 & $94.9 \%)$; Total dropped users: $5.1 \%$ \\
\hline \multirow[b]{2}{*}{ 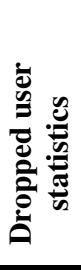 } & Reason & No. of users & $\%$ of users (of the $5.1 \%$ / of the total) \\
\hline & $\begin{array}{l}\text { Mobile ERP limit } \\
\text { Noise rise limit } \\
\text { Primary CE limit } \\
\text { CPICH power limit } \\
\text { DPCH power limit } \\
\text { Code limit }\end{array}$ & $\begin{array}{c}0.1 \\
0 \\
0 \\
3.9 \\
31.7 \\
0\end{array}$ & $\begin{array}{c}0.4 / 0.02 \\
0 \\
0 \\
10.8 / 0.55 \\
88.8 / 4.53 \\
0\end{array}$ \\
\hline \multirow[b]{2}{*}{ 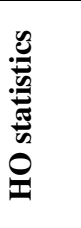 } & Type of HO & No. of users & $\%$ of users \\
\hline & $\begin{array}{l}\text { Not in HO } \\
\text { Softer HO } \\
\text { Soft HO } \\
\text { Soft-soft HO } \\
\text { Softer-soft HO }\end{array}$ & $\begin{array}{c}160.3 \\
2.2 \\
124.4 \\
365.6 \\
11.2\end{array}$ & $\begin{array}{c}24.1 \\
0.3 \\
18.7 \\
55.1 \\
1.8\end{array}$ \\
\hline
\end{tabular}


Table 8. Simulation report with changes in the power limits of $\mathrm{DPCH}$ and $\mathrm{CPICH}$

\begin{tabular}{|c|c|c|c|}
\hline \multicolumn{4}{|c|}{ User type: Voice and CS data at $64 \mathrm{kbps}$} \\
\hline & Jsers attempted: 700.9 & sers served: 67 & $96.6 \%)$; Total dropped users: $3.4 \%$ \\
\hline \multirow[b]{2}{*}{ 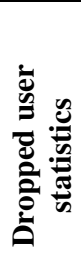 } & Reason & No. of users & $\%$ of users (of the $3.4 \%$ / of the total) \\
\hline & $\begin{array}{l}\text { Mobile ERP limit } \\
\text { Noise rise limit } \\
\text { Primary CE limit } \\
\text { CPICH power limit } \\
\text { DPCH power limit } \\
\text { Code limit }\end{array}$ & $\begin{array}{c}0.2 \\
0 \\
0 \\
5.2 \\
18.6 \\
0\end{array}$ & $\begin{array}{c}0.8 / 0.03 \\
0 \\
0 \\
21.8 / 0.74 \\
77.4 / 2.63 \\
0\end{array}$ \\
\hline \multirow[b]{2}{*}{ 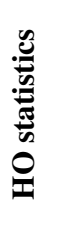 } & Type of HO & No. of users & $\%$ of users \\
\hline & $\begin{array}{l}\text { Not in HO } \\
\text { Softer HO } \\
\text { Soft HO } \\
\text { Soft-soft HO } \\
\text { Softer-soft HO }\end{array}$ & $\begin{array}{c}165.5 \\
2.2 \\
131.1 \\
366.0 \\
12.0\end{array}$ & $\begin{array}{c}24.5 \\
0.3 \\
19.4 \\
54.1 \\
1.8\end{array}$ \\
\hline
\end{tabular}

While it was not possible to solve the power sharing problem by changing the layout of the BSs, the authors tried to perform new simulations after changing the limits of the power dedicated to DPCH and CPICH. As reported in Table 8, it is possible to partially improve the performances: the system can now attend $96.6 \%$ of the users. We could try to tune these power limits further, but this would not be enough to solve the problems inherent to sharing a common resource between too many users and load. While providing the possibility for one user's signal to be recovered from different receivers in the UL, macrodiversity creates an overload in the DL since more than one BS has to provide a part of its limited power to just one user.

\section{Conclusions}

The problem of distributing UMTS base stations in a densely populated area was addressed through simulation analysis in a realistic scenario (the city of Barcelona, Spain). The LB was computed and checked against the single-cell case with excellent results. This is useful to validate the simulator against the model and to gain confidence in the simulation results that follow.

To illustrate the trial-and-error process needed in this kind of planning, a first distribution of BSs following the classical hexagonal pattern was evaluated. Several problems in coverage (i.e. shadows) and capacity were observed. The second layout introduces slight changes to the first and improves coverage. However, capacity results are very difficult to improve in this process. It is foreseen that this trend will not improve even if more trials are carried out.

\section{References}

1. Holma, H., Toskala, A.: WCDMA for UMTS Radio Access for 3rd Generation Mobile Communications. John Wiley \& Sons (2002)

2. Dehghan, S., Lister, D., Owen, R., Jones, P.: W-CDMA Capacity and Planning Issues. Electronics and Communication Engineering Journal, Vol. 12, Issue 3 (2000) 101-118

3. Zola, E., Barceló, F., Martín, I.: Simulation Analysis of Cell Coverage and Capacity Planning in a UMTS Environment: A Case Study. IASTED International Conference Communication Systems and Networks (2003) 342-346

4. Veeravalli, V.V., Sendonaris, A.: The Coverage-Capacity Tradeoff in Cellular CDMA Systems. IEEE Trans. on Vehicular Technology, Vol. 48, No 5 (1999) 1443-1450 
5. Coinchon, M., Salovaara, A., Wagen, JF.: The Impact of Radio Propagation Predictions on Urban UMTS Planning. International Zurich Seminar on Access, Transmission and Networking (2002) 32-1 - 32-6

6. Schroder, B., Liesenfeld, B., Weller, A., Leibnitz, K., Staehle, D., Phuoc Tran-Gia: An Analytical Approach for Determining Coverage Probabilities in Large UMTS Networks. IEEE 54th Vehicular Technology Conference, VTC 01 Fall (2001) 1750 -1754

7. Hata, M.: Empirical Formula for Propagation Loss in Land Mobile Radio Service. IEEE Trans. on Vehicular Technology, Vol. 29, No. 3 (1980)

8. Laiho, J., Wacker, A., Novosad, T.: Radio Network Planning and Optimisation for UMTS. John Wiley and Sons, 2001

9. Owen, R., Burley, S., Jones, P., Messer, V.: Considerations for UMTS Capacity and Range Estimation. IEEE Colloquium on Capacity and Range Enhancement Techniques for the Third Generation Mobile Communications and Beyond, Ref. No. 2000/003 (2000) 5/1 - 5/6

10. Pattuelli, R., Zingarelli, V.: Precision of the estimation of area coverage by planning tools in cellular systems. IEEE Personal Communications, Vol. 7, Issue 3 (2000) 50 -53

11. Garg, V., Yellapantula, R.: A Tool to Calculate Erlang Capacity of a BTS Supporting 3G UMTS System. IEEE International Conference on Personal Wireless Communications (2000) $173-177$ 